\title{
CARACTERIZAÇÃO EPIDEMIOLÓGICA DE PACIENTES ADULTOS INTERNADOS EM UMA UNIDADE DE TERAPIA INTENSIVA DE QUEIMADOS*
}

\author{
Marcia Bernadete Camuci', Júlia Trevisan Martins², Alexandrina Aparecida Maciel Cardeli², \\ Maria Lúcia do Carmo Cruz Robazzi ${ }^{3}$
}

\begin{abstract}
RESUMO: : Estudo quantitativo, exploratório, descritivo e retrospectivo objetivou identificar o perfil epidemiológico de adultos internados em Unidade de Terapia Intensiva quanto às variáveis sociodemográficas e características da queimadura, a partir de dados coletados de 50 prontuários, de outubro de 2011 a maio de 2012. Houve predomínio do sexo masculino (74\%); a média de idade foi de 41,02 anos; a queimadura térmica ocorreu em $88 \%$, prevalecendo os acidentes domésticos caracterizados por escaldo; uso de álcool para acender a churrasqueira ou fogão à lenha; explosão de panela, botijão de gás, fogos de artifício; e incêndio no domicílio. As queimaduras de terceiro grau ocorreram em $70 \%$ dos casos, a lesão inalatória foi identificada em $22 \%$ dos pacientes, e a alta prevaleceu sobre o óbito como desfecho. Conclui-se que a maioria das queimaduras pode ser prevenida; é necessário implementar políticas públicas e ações de educação em saúde continuada para a sociedade. DESCRITORES: Queimaduras; Terapia intensiva; Unidades de queimados; Políticas públicas.
\end{abstract}

\section{CARACTERIZACIÓN EPIDEMIOLÓGICA DE PACIENTES ADULTOS INTERNADOS EN UNIDAD DE TERAPIA INTENSIVA DE QUEIMADOS}

RESUMEN: Estudio cuantitativo, exploratorio, descriptivo y retrospectivo cuya finalidad fue identificar el perfil epidemiológico de adultos internados en Unidad de Terapia Intensiva cuanto a las variables sociodemográficas y características de la quemadura, con base en datos obtenidos de 50 prontuarios, de octubre de 2011 a mayo de 2012 . Hubo predominio del sexo masculino (74\%); la media de edad fue de 41,02 años; la quemadura térmica ocurrió para encender la "churrasquera" o fogón a leña; explosión de olla, bombona de gas, fuegos artificiales; e incendio en domicilio. Las quemaduras de tercer grado ocurrieron en $70 \%$ de los casos, la lesión por inhalación fue identificada en $22 \%$ de los pacientes, y el alta prevaleció sobre el óbito como desenlace. Se concluye que la mayoría de las quemaduras puede ser prevenida; así que es necesario implementar políticas públicas y acciones de educación en salud continuada para la sociedad.

DESCRIPTORES: Quemaduras; Terapia intensiva; Unidades de quemados; Políticas públicas.

\section{EPIDEMIOLOGICAL CHARACTERIZATION OF ADULT PATIENTS HOSPITALIZED IN A BURNS INTENSIVE CARE UNIT*}

\begin{abstract}
This quantitative, exploratory, descriptive and retrospective study aimed to identify the epidemiological profile of adults hospitalized in an intensive care unit in relation to the socio-demographic variables and the characteristics of the burn, based on data collected from 50 hospital records, from October 2011 to May 2012. The male sex predominated (74\%); the mean age was 41.02 years; thermal burns occurred in $88 \%$ of cases, with the following predominating: domestic accidents, characterized by scalds; the use of alcohol for lighting barbecues or wood-burning stoves; the explosion of pressure cookers, gas canisters, and fireworks; and fires in the home. Third degree burns occurred in $70 \%$ of the cases, inhalation injuries were identified in $22 \%$ of the patients, and discharge from hospital was a more common outcome than death. It is concluded that the majority of burns can be prevented; it is necessary to implement public policies and continuous health education actions for society.
\end{abstract}

DESCRIPTORS: Burns; Intensive care; Burns centers; Public policies.

\footnotetext{
*Este artigo foi desenvolvido a partir de Dissertação de Mestrado intitulada "Carga de trabalho de Enfermagem de uma Unidade de Terapia Intensiva de Queimados segundo o Nursing Activities Score", apresentada ao Mestrado em Enfermagem da Universidade Estadual de Londrina, em 2012.
}

${ }^{1}$ Enfermeira. Mestranda em Enfermagem. Universidade Estadual de Londrina. Londrina-PR-Brasil ${ }^{2}$ Enfermeira. Doutora em Enfermagem. Professora da Universidade Estadual de Londrina. Londrina-PR-Brasil ${ }^{3}$ Enfermeira. Doutora em Enfermagem. Professora da Universidade de São Paulo. Ribeirão Preto-SP-Brasil 


\section{INTRODUÇÃO}

Estima-se que 195.000 mortes/ano são causadas por queimaduras no mundo e a grande maioria ocorre em países de baixa e média renda. Nos países de menor renda a morte de crianças por queimadura é sete vezes maior do que nos países de alta renda ${ }^{(1)}$.

A queimadura é um grande problema para a saúde pública brasileira, porque além de causar elevado número de mortes todos os anos provoca sequelas físicas e psicológicas que marcam suas vítimas, e são altamente onerosas ${ }^{(2)}$. $\mathrm{O}$ valor médio gasto pelo Ministério da Saúde (MS) no Brasil, com internação de pessoas com queimaduras graves, é de um milhão de reais/mês ${ }^{(3)}$, cerca de 500 mil dólares/mês.

A Sociedade Brasileira de Queimaduras (SBQ) afirma que um milhão de pessoas são vítimas de queimaduras a cada ano e entre 1996 e 2008 foram registradas 13.735 mortes causadas por este problema $^{(4)}$. Problemas sociais e financeiros ligados ao trabalho por afastamento, aposentadorias e reabilitações são alguns dos transtornos causados pelas queimaduras. Esses agravos atingem também psicologicamente os pacientes e seus familiares, não só pelas inúmeras cicatrizes e deformidades físicas, mas também pelo longo tempo de internação que, muitas vezes, exigem ${ }^{(2)}$.

A queimadura é uma ferida traumática causada, principalmente, por agentes térmicos, químicos, elétricos ou radioativos. Acontece nos tecidos de revestimentos do corpo humano, podendo destruir parcial ou totalmente a pele e seus anexos, e também as camadas mais profundas, como tecido celular subcutâneo, músculos, tendões e ossos ${ }^{(1,5)}$.

Frente ao exposto, o presente trabalho justifica-se, pois, estudos desta natureza, podem contribuir para orientação de políticas públicas e ações de educação em saúde para prevenção de queimaduras, visto que possibilita identificar características específicas, sexo, faixa etária, tipo de lesão, etiologia, agente causal, classificação, dentre outros. Conhecer a população atendida se configura como alicerce para os gestores da Unidade de Tratamento Intensiva (UTI) do Centro de Tratamento de Queimado (CTQ) estudada, para o aprimoramento do serviço, desde a assistência aos pacientes e familiares, educação em serviço, treinamento dos funcionários, bem como desenvolvimento de projetos preventivos para a população.

Assim, este estudo teve como objetivo identi- ficar o perfil epidemiológico de pacientes adultos internados na UTI de um CTQ quanto às variáveis sociodemográficas e características da queimadura.

\section{MÉTODO}

Trata-se de um estudo exploratório, descritivo, retrospectivo, com abordagem quantitativa, realizado na UTI do CTQ do Hospital Universitário de Londrina, composta por seis leitos. Ressalta-se que no Estado do Paraná só existem dois CTQ, um localizado na cidade de Curitiba e outro na cidade de Londrina.

O período de coleta foi de outubro de 2011 a maio de 2012, utilizando-se dados extraídos de 50 prontuários de todos os pacientes adultos admitidos neste período e que atenderam aos critérios de inclusão: ter idade igual ou superior a 18 anos, e que permaneceram internados por um período mínimo de 24 horas. As readmissões foram excluídas do estudo.

Para a coleta de dados foi utilizado um instrumento contendo as seguintes variáveis: sexo, idade, etiologia da queimadura (térmica, química, elétrica, radiação, outras), agente (álcool), profundidade da lesão $\left(2^{\circ}\right.$ e $3^{\circ}$ grau), lesão inalatória, classificação (acidente doméstico, acidente de trabalho, suicídio, homicídio), procedência, desfecho (alta, óbito). Os dados foram organizados e armazenados em planilha eletrônica Excel 2007 e para análise dos dados foi utilizado o programa Statistical Package of Social Sciences (SPSS) ${ }^{\circledR}$, versão 19.0.

O projeto foi aprovado pelo Comitê de Ética em Pesquisa da Universidade Estadual de Londrina (UEL), com parecer favorável sob o número 214/2011 e CAAE 0187.0.268.000-11.

Destaca-se que para realização deste estudo, foi preenchido e assinado o "Termo de Confidencialidade e Sigilo", no qual a pesquisadora assumiu o compromisso de manter confidencialidade sobre todas as informações técnicas e outras relacionadas ao projeto de pesquisa, inclusive o respeito à identidade dos pesquisados.

\section{RESULTADOS}

A Tabela 1 mostra as características sociodemográficas dos pacientes e das queimaduras. Houve predomínio de pacientes do sexo masculino (74\%) em relação ao feminino (26\%) e a idade variou entre $18 \mathrm{e}$ 76 anos, com uma média de 41,02 anos. A queimadura 
térmica ocorreu numa frequência de $88 \%$ e a elétrica em $12 \%$; as queimaduras de terceiro grau ocorreram em $70 \%$ dos casos e a lesão inalatória ocorreu em $22 \%$ dos pacientes. A alta da UTI dos pacientes prevaleceu sobre os óbitos e representaram 74\%. Identificou-se o predomínio dos acidentes domésticos caracterizados por escaldo, uso de álcool para acender a churrasqueira, uso de álcool para acender fogão a lenha, explosão de panela, explosão de gás, explosão de fogos de artifício e incêndio no domicílio.

No que se refere aos dias de internação identificou-se que variaram de dois a 97 dias, apresentando uma média de 24 dias.

Tabela 1 - Distribuição dos pacientes adultos com queimaduras, segundo as variáveis sociodemográficas e características das queimaduras. Londrina-PR-Brasil, 2012

\begin{tabular}{|c|c|c|}
\hline Variáveis & $\begin{array}{c}\mathbf{n} \\
50\end{array}$ & $\begin{array}{c}\% \\
100\end{array}$ \\
\hline \multicolumn{3}{|l|}{ Sexo } \\
\hline Masculino & 37 & 74 \\
\hline Feminino & 13 & 26 \\
\hline \multicolumn{3}{|l|}{ Faixa Etária } \\
\hline$<20$ & 02 & 4 \\
\hline $20-29$ & 11 & 22 \\
\hline $30-39$ & 14 & 28 \\
\hline $40-49$ & 08 & 16 \\
\hline $50-59$ & 07 & 14 \\
\hline 60 e mais & 08 & 16 \\
\hline \multicolumn{3}{|c|}{ Etiologia da queimadura } \\
\hline Térmica & 44 & 88 \\
\hline Elétrica & 06 & 12 \\
\hline \multicolumn{3}{|c|}{ Agente causador (álcool) } \\
\hline Sim & 25 & 50 \\
\hline Não & 25 & 50 \\
\hline \multicolumn{3}{|c|}{ Profundidade da lesão } \\
\hline Segundo Grau & 15 & 30 \\
\hline Terceiro Grau & 35 & 70 \\
\hline \multicolumn{3}{|l|}{ Lesão inalatória } \\
\hline Sim & 11 & 22 \\
\hline Não & 39 & 78 \\
\hline \multicolumn{3}{|l|}{ Desfecho } \\
\hline Alta da UTI & 37 & 74 \\
\hline Óbito & 13 & 26 \\
\hline \multicolumn{3}{|c|}{ Classificação da queimadura } \\
\hline Acidente doméstico & 32 & 64 \\
\hline Acidente de trabalho & 10 & 20 \\
\hline Homicídio & 05 & 10 \\
\hline Suicídio & 03 & 6 \\
\hline
\end{tabular}

\section{DISCUSSÃO}

Os dados deste estudo demonstraram o predomínio dos pacientes do sexo masculino, também verificado em estudo que analisou o perfil epidemiológico de pacientes internados em um CTQ na cidade do Rio de Janeiro $^{(6)}$ e no Hospital do Servidor Público Estadual de São Paulo ${ }^{(7)}$. As internações de pacientes adultos com queimaduras encontrados no presente estudo mostraram o predomínio em uma faixa etária que é semelhante aos dados de um estudo desenvolvido no CTQ da Universidade Federal de São Paulo no qual $33,6 \%$ dos pacientes tinham idade entre 31 a 50 anos e $31,7 \%$ idade entre 19 a 30 anos $^{(8)}$.

Os indivíduos que compõem esta faixa etária fazem parte da população economicamente ativa, gerando problemas de ordem econômica e social. As queimaduras, geralmente, atingem indivíduos em idade produtiva, que muitas vezes são responsáveis pela única fonte de renda em seu domicílio( ${ }^{(7)}$. Em muitos casos as queimaduras provocam danos permanentes como redução da capacidade funcional, sequelas físicas e estéticas que podem se transformar também em traumas psicológicos, acarretando em condição crônica de saúde ${ }^{(9)}$.

Durante o período desta investigação não ocorreram casos de queimaduras químicas ou por radiação. Houve o predomínio de queimaduras térmicas, sendo que metade destas foi causada pelo uso de álcool líquido e fogo. Esses dados são semelhantes à investigação que avaliou o perfil epidemiológico e antropométrico de pacientes internados em um CTQ em Brasília e identificou que o álcool associado ao fogo foi o agente causal mais prevalente ${ }^{(10)}$. Infere-se que estes dados podem estar relacionados com uso indiscriminado deste produto, principalmente nos domicílios, pelo fácil acesso da população em adquiri-lo e pelo desconhecimento dos riscos de acidentes. Assim sendo, é fundamental que os profissionais de saúde realizem orientações aos pacientes, familiares e sociedade em geral sobre os perigos e cuidados que se deve ter ao utilizar a combinação álcool líquido e fogo.

Constatou-se, neste estudo, que a queimadura elétrica ocorreu numa frequência bem menor que a térmica. Dados esses que são semelhantes ao estudo realizado no CTQ do Hospital Federal do Andaraí que mostrou que apenas $9 \%$ do total de 1.773 internações eram de etiologia elétrica ${ }^{(11)}$. A baixa frequência desta etiologia não é menos preocupante, autores ${ }^{(11-12)}$ afirmam que a queimadura por corrente de baixa tensão apresenta pequena taxa de morbidade e mortalidade, com raras 
internações, porém as geradas por alta tensão, maiores que 1000 volts, são de grande gravidade para a vítima. Enfatiza-se que as sequelas causadas pela queimadura oriunda por alta tensão pode causar traumas físicos e psicológicos, dificuldades para caminhar, amputações que muitas vezes exigem o uso de próteses, cicatrizes, lesões neurológicas permanentes, além do aspecto estético do paciente e distorção da própria imagem ${ }^{(11-12)}$.

As queimaduras de terceiro grau ocorreram em $70 \%$ dos casos, fato que se diferenciou dos resultados encontrados, no CTQ do hospital de urgência de Sergipe em que $15,25 \%$ dos pacientes internados, apresentaram queimaduras de terceiro grau, $83,86 \%$ de segundo grau e $0,9 \%$ de primeiro grau ${ }^{(13)}$. Sabe-se que existe certa dificuldade em se obter dados precisos sobre a profundidade das lesões, pois alguns profissionais atenta-se em anotar no prontuário apenas as queimaduras de profundidades mais graves, mesmo quando existem as de profundidades diferentes em um mesmo paciente ${ }^{(14)}$. Assim, é imprescindível desenvolver ações de educação que mostrem aos profissionais de saúde a importância de anotar todos os dados dos pacientes, visto que, trata-se de registro legal, é uma fonte de informação clínica e administrativa para tomada de decisão, bem como meio de comunicação compartilhado entre todos os profissionais.

A lesão inalatória neste estudo ocorreu em $22 \%$ dos pacientes. Tais dados apresentam diferença em relação ao estudo que indicou que $10,9 \%$ do total das internações de queimados foram por lesão inalatória ${ }^{(10)}$, esta foi a principal responsável pela mortalidade (60-80\%) dos pacientes que sofreram queimadura ${ }^{(15)}$. Esta lesão apresenta alto índice de mortalidade e complexidade no tratamento, produz importante resposta inflamatória, com repercussões pulmonares e sistêmicas, o que demonstra a gravidade deste tipo de queimadura ${ }^{(16)}$.

A alta da UTI do CTQ prevaleceu no presente. Resultados diferentes foram encontrados em investigação realizada para demonstrar o perfil dos pacientes atendidos na UTQ na cidade de Aracaju, na qual a taxa de óbito foi $3,1 \%{ }^{(17)}$. Porém, índice elevado também foi encontrado num estudo de um CTQ da Alemanha onde a taxa de óbito foi de $15 \%{ }^{(18)}$.

Sugere-se que a alta taxa de mortalidade encontrada neste estudo possa estar relacionada ao fato de que grande número dos pacientes admitidos na UTI do CTQ do HUL são provenientes de cidades do interior do estado do Paraná e haviam sofrido a queimadura há alguns dias. Esse fato pode contribuir para a demora em iniciar a terapia específica, o que pode trazer sé- rias consequências e complicações para o sucesso do tratamento. Tal constatação deve orientar a equipe de saúde no planejamento dos cuidados para evitar complicações e agravamentos no estado geral dos pacientes. Porém, é fato que a ressuscitação volêmica adequada iniciada logo após o acidente representa um ganho importante na sobrevida dos pacientes vítimas de grandes queimaduras ${ }^{(19)}$. Assim, é preciso desenvolver treinamentos para os profissionais de saúde no que tange aos primeiros cuidados a serem prestados aos pacientes com queimaduras.

Outros fatores que contribuíram para o óbito foram à alta incidência de paciente com lesão inalatória, a Superfície Corporal Queimada e a idade. Sabe-se que a presença de lesão inalatória aumenta em $20 \%$ a mortalidade associada à extensão da queimadura ${ }^{(16)}$. A idade, a extensão da superfície corpórea queimada e presença de lesão inalatória são variáveis que podem interferir em graus variados com a ocorrência de óbito ${ }^{(20)}$. É provável, então, que estas variáveis contribuíram para a elevada taxa de mortalidade observada no presente estudo.

No que concerne aos acidentes domésticos infere-se que a maioria poderia ter sido evitado se as pessoas estivessem cientes dos riscos a que estão expostos diante de fatores como: acender churrasqueira e fogão a lenha com álcool, descuido com os líquidos quentes, uso de fogos de artifício, dentre outros. Em estudo realizado em um Hospital Escola do Noroeste do Paraná, os resultados demonstraram que os acidentes domésticos foram os mais frequentes em relação aos outros tipos de acidentes por queimaduras ${ }^{(21)}$. Resultados semelhantes foram identificados em estudo epidemiológico no qual $45,7 \%$ das queimaduras dos pacientes ocorreram no domicílio ${ }^{(22)}$.

No que se refere aos dias de internação, dados análogos a do presente estudo foi encontrado em investigação realizada em um CTQ de Brasília, onde o tempo médio de internação foi de 27 dias $^{(10)}$.

Por fim, ressalta-se que os achados deste estudo mostraram que os objetivos traçados foram alcançados, porém apresenta limitações por tratar-se de estudo retrospectivo onde variáveis como ocupação antes do trauma, sequelas, dentre outras, não foram possíveis de identificar. Assim sendo, sugere-se a realização de estudos que abordem essas limitações.

\section{CONCLUSÃo}

Houve predomínio de pacientes do sexo masculino, com idade média de 41,02 anos, que é tida como idade produtiva. A queimadura térmica ocorreu numa 
frequência maior do que a elétrica, assim como as queimaduras de terceiro grau. A lesão inalatória esteve presente em $22 \%$ dos pacientes; a alta dos pacientes prevaleceu sobre os óbitos e houve predomínio dos acidentes domésticos.

Os resultados do presente estudo apontam para necessidade de intervenções no campo da Educação em Saúde da população, com a finalidade de realizar ações de prevenção e promoção saúde, porém não só na atenção primária, mas também na atenção secundária e terciária, como é o caso dos serviços especializados em tratamento de pacientes com queimaduras, ou seja, as UTI dos CTQ.

\section{REFERÊNCIAS}

1. WHO. World Health Organization. Fact sheet. Burns, Guildford, n. 365, 2012. [Internet] 2012 (acesso em 06 jul 2012). Disponível: http://www.who.int/mediacentre/ factsheets/fs365/en/.

2. Takejima ML, Netto RFB, Toebe BL, Andretta MA, Prestes MA, Takaki JL, et al. Prevenção de queimaduras: avaliação do conhecimento sobre prevenção de queimaduras em usuários das unidades de saúde de Curitiba. Rev Bras Queimaduras. 2011;10(3):85-8.

3. Ministério da Saúde(BR). Informe saúde. Queimaduras. Ano VI. No 152. 2002. [Internet] 2002 (acesso em 29 jul 2012). Disponível: http://bvsms.saude.gov.br/bvs/ periodicos/informesaude/informe152.pdf.

4. Sociedade Brasileira de Queimaduras, 2012. [Internet] 2012 (acesso em 29 jul 2012]. Disponível: http://www. sbqueimaduras.com.br/sbq/?s=QUEIMADURAS+LE GISLA $\%$ C3\%87\%C3\%83O.

5. Serra MCVF, Gomes DR, Crisóstomo MR. Fisiologia e fisiopatologia. In: Maciel E, Serra MC. Tratado de queimaduras. São Paulo: Atheneu; 2006. p.37-42.

6. Leão CEG, Andrade ES, Fabrini DS, Oliveira RA, Machado GLB, Gontijo LC. Epidemiologia das queimaduras no estado de Minas Gerais. Rev. Bras. Cir. Plást. 2011;26(4):573-7.

7. Souza AA, Mattar CA, Almeida PCC, Faiwichow L, Fernandes FS, Neto ECA, et al. Perfil epidemiológico dos pacientes internados na Unidade de Queimaduras do Hospital do Servidor Público Estadual de São Paulo. Rev Bras Queimaduras. 2009;8(3):87-90.

8. Lacerda LA, Carneiro AC, Oliveira AF, Gragnani A,
Ferreira LM. Estudo epidemiológico da Unidade de Tratamento de Queimaduras da Universidade Federal de São Paulo. Rev Bras Queimaduras. 2010;9(3):82-8.

9. Costa M, Rossi L, Dantas R, Trigueros L. Imagem corporal e satisfação no trabalho entre adultos em reabilitação de queimaduras. Cogitare enferm. 2010;15(2):209-16.

10. Lima LS, Araújo MAR, Cavendish TA, Assis EM, Aguiar G. Perfil epidemiológico e antropométrico de pacientes internados em uma unidade de tratamento de queimados em Brasília, Distrito Federal. Com. Ciências Saúde. 2010;21(4):301-8.

11. Souza AL, Oliveira BCE, Andrade C, Monteso K, Rebelo PG, Rodrigues RPC, et al. Queimadura elétrica no Hospital Federal do Andaraí de 1997 a 2010: análise de 152 casos. Rev Bras Queimaduras. 2012;11(2):80-4.

12. Leonardi DF, Laporte GA, Tostes FM. Amputação de membro por queimadura elétrica de alta voltagem. Rev Bras Queimaduras. 2011;10(1):27-9.

13. Reis IF, Moreira CA, Costa ACSM. Estudo epidemiológico de pacientes internados na unidade de tratamento de queimados do hospital de urgência de Sergipe. Rev Bras Queimaduras. 2011;10(4):114-8.

14. Mendes CA, Sá DM, Padovese SM, Cruvinel SS. Estudo epidemiológico de queimaduras atendidas nas Unidades de Atendimento Integrado de Uberlândia-MG entre 2000 a 2005. Rev Bras Queimaduras. 2009;8(1):18-22.

15. Sauge JM, Souza R, Jardim C. Efeito da queimadura sobre o pulmão. In: Maciel E, Serra MC. Tratado de queimaduras. São Paulo: Atheneu; 2006. p. 97-102.

16. Spinelli J, Rezegue L, Fiorin R, Bragança KR. Lesão inalatória grave: tratamento precoce e reversão do quadro. Relato de caso e revisão de literatura. Rev Bras Queimaduras. 2010;9(1):31-4.

17. Moreira CA, Reis IF, Costa ACSM. Perfil epidemiológico de pacientes atendidos na unidade de tratamento de queimados no município de Aracaju (SE). Cadernos de Graduação - Ciências Biológicas e da Saúde. 2011;13(14):111-27.

18. Theodorou $\mathrm{P}, \mathrm{Xu} \mathrm{W}$, Weinand $\mathrm{C}$, Perbix W, Maegele $\mathrm{M}$, Lefering $\mathrm{R}$, et al. Incidence and treatment of burns: A twenty-year experience from a single center in Germany. Burns. 2013;39:49-54.

19. Cunha NT. Complicações das queimaduras In: Maciel 
E, Serra MC. Tratado de queimaduras. São Paulo: Atheneu; 2006. p. 81-90.

20. Rosa DMC. Epidemiologia, lesão inalatória e biomarcadores: relação com prognóstico de adultos queimados internados em uma unidade de referência (tese). Botucatu (SP): Faculdade de Medicina de Botucatu; 2009. 153 p.

21. Balan MAJ, Oliveira MLF, Trass G. Características das vítimas de queimaduras atendidas em unidade de emergência de um Hospital Escola do Noroeste do Paraná. Ciênc. cuid. saúde. 2009;8(2):169-75.

22. Montes SF, Barbosa MH, Souza Neto AL. Aspectos clínicos e epidemiológicos de pacientes queimados internados em um Hospital de Ensino. Rev Esc Enferm USP. 2011;45(2):369-73. 